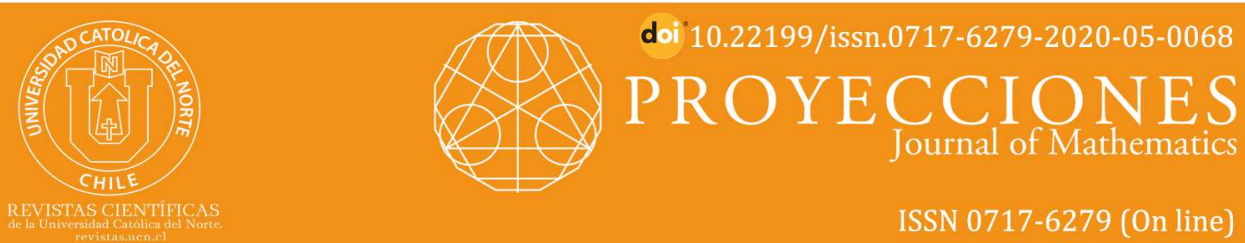

\title{
The nonsplit domination in subdivision graph
}

R. Jemimal Chrislight ${ }^{1}$ 응 orcid.org/0000-0002-1673-2076

Y. Therese Sunitha Mary² (1) orcid.org/0000-0002-5182-9631

${ }^{1}$ St. Xavier's College (Autonomous)*, PG and Research, Dept. of Mathematics, Palymkottai,

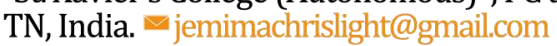

${ }^{2}$ St. Xavier's College (Autonomous)*, Dept. of Mathematics, Palayamkottai, TN, India.

sunithanepolian@gmail.com

Received: August 2018 | Accepted: February 2020

\section{Abstract:}

A dominating set $D$ of a graph $G=(V, E)$ is a nonsplit dominating set if the induced subgraph $\langle V-D\rangle$ is connected. The nonsplit domination number $\gamma_{n s}(G)$ of $G$ is the minimum cardinality of a nonsplit dominating set. An edge $e=u v$ of a graph $G$ is said to be subdivided if e is replaced by the edges $u w$ and $v w$ for some vertex $w$ not in $V(G)$. The graph obtained from $G$ by subdividing each edge of $G$ exactly once is called the subdivision graph of $G$ and is denoted by $S(G)$. In this paper, we study the nonsplit domination number of subdivision graph. We determine exact values of the nonsplit domination number of subdivision graph for some standard graphs. We also obtain bounds and relationship with other graph theoretic parameters for the $\gamma_{n s}(S(G))$.

Keywords: Domination number; Nonsplit domination number; Subdivision graph; Nonsplit domination number of subdivision graph.

MSC (2020): $05 C 69$.

Cite this article as (IEEE citation style):

R. J. Chrislight and Y. T. Sunitha Mary, "The nonsplit domination in subdivision graph", Proyecciones (Antofagasta, On line), vol. 39, no. 5, pp. 1113-1120, Oct. 2020, doi: 10.22199/issn.07176279-2020-05-0068.

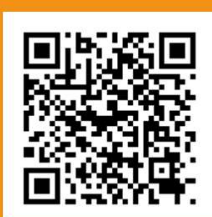

Article copyright: (c) 2020 R. Jemimal Chrislight and Y. Therese Sunitha Mary. This is an open access article distributed under the terms of the Creative Commons License, which permits unrestricted use and distribution provided the original author and source are credited.

(cc) BY

*Affiliated to Manonmaniam Sundaranar University, Abishekapatti, Tirunelveli, TN, India. 


\section{Introduction}

In this paper,the graphs considered here are finite, undirected nontrivial and connected without loop or multiple edges. Any undefined term in this paper may be found in $[1,2]$ and the graph $G$ has $p$ vertices and $q$ edges.

Let $G=(V, E)$ be a graph. A set $D$ subset of $V$ is a dominating set of $G$ if every vertex in $V-D$ is adjacent to some vertex in $D$. The domination number $\gamma(G)$ of $G$ is the minimum cardinality of a dominating set. The open neighborhood of a vertex $v$ in the graph $G$ is the set of vertices adjacent to $v$. It is denoted by $N(v)$ and the closed neighborhood of $v$ is $N[v]=N(v) \cup v$.

The concept of nonsplit domination has been studied by V. R. Kulli and B. Janakiram [4]. A dominating set $D$ of a graph $G=(V, E)$ is a nonsplit dominating set if the induced graph $\langle V-D\rangle$ is connected. The nonsplit domination number $\gamma_{n s}(G)$ is the minimum cardinality of a non- split dominating set. For example, a $\gamma_{n s}$-set in $G$ represents a minimum set of transformers that can communicate directly with other vertices which are consider as post. But every post is must adjacent to at least one trans- former. The concept of nonsplit domination is used to find the minimum possibilities to cover all the post and make 24/7 electric supply in the city. Also, it has applied in communication network, transport, railway, land surveying, etc.

The behavior of a network in the presence of a fault can be analyzed by determining the effect that removing the edge and adding new vertex along with two new edges. The subdivision graph is studied in [1], [2], [3]. An edge $e=u v$ of a graph $G$ is said to be subdivided if $e$ is replaced by the edges $u w$ and $v w$ for some vertex $w$ not in $V(G)$. The subdivision graph obtained from $G$ by subdividing each edge of $G$ and is denoted by $S(G)$.

We need the following theorems in [4].

Theorem 1.1. For any cycle $C_{p}, \gamma_{n s}\left(C_{p}\right)=p-2$.

Theorem 1.2. For any complete graph $K_{p}$ with $p \geq 2$ vertices, $\gamma_{n s}\left(K_{p}\right)=$ 1.

Theorem 1.3. For any path $P_{p}$ with $p \geq 4$ vertices, $\gamma_{n s}\left(P_{p}\right)=p-2$. 
Theorem 1.4. For any graph $G, \gamma_{n s}(G) \leq p-\omega(G)+1$ where $\omega(G)$ is the clique number of $G$.

In this paper, we determine exact values of the nonsplit domination number of subdivision graph for some standard graphs. We also obtain bounds and relationship with other graph theoretic parameters for the nonsplit domination number of subdivision graph.

\section{Main Results}

The following are immediate from Theorem 1.1 and Theorem 1.3 respectively.

Proposition 2.1. (i) For any cycle $C_{p}, \gamma_{n s}\left(S\left(C_{p}\right)\right)=2 p-2$. (ii) For any path $P_{p}$ with $p \geq 3$ vertices, $\gamma_{n s}\left(S\left(P_{p}\right)\right)=2 p-3$.

Theorem 2.2. For any star $K_{1, p-1}, \gamma_{n s}\left(S\left(K_{1, p-1}\right)\right)=p$.

Proof. Let $G$ be any star and $S\left(K_{1, p-1}\right)$ is healthy spider. Let $V(G)=$ $\left\{v_{0}, v_{1}, v_{2}, \ldots, v_{p-1}\right\}$ be the verties of $G$ and $W=\left\{w_{1}, w_{2}, \ldots, w_{q}\right\}$ be the vertices in $S(G)$ which subdivides the edges of $G$. Clearly, $D$ contains all the end vertices of $S(G)$, say $n=\left\{v_{1}, v_{2}, v_{3}, \ldots, v_{p-1}\right\}$.

Now, $S(G)-n$ is a star. Then $D$ contains at least one vertex in a star for some $w_{i}(1 \leq i \leq q)$ of $S(G)$. So that, the nonsplit dominating set $D$ of $S(G)$ is $\left\{v_{1}, v_{2}, v_{3}, \ldots, v_{p-1}\right\} \cup\left\{w_{i}\right\}$ for some $w_{i}, 1 \leq i \leq q$.

$$
\begin{aligned}
|D| & =p-1+1 \\
& =p .
\end{aligned}
$$

Theorem 2.3. For any wheel graph $W_{p}$ with $p \geq 4, \gamma_{n s}\left(S\left(W_{p}\right)\right)=q+1$.

Proof. Let $\mathrm{G}$ be a wheel graph. Let $V(G)=\left\{v_{0}, v_{1}, v_{2}, \ldots, v_{p-1}\right\}$ be the vertices of $G$. Let $W=\left\{w_{1}, w_{2}, w_{3}, \ldots, w_{p-1}, w_{p}, \ldots, w_{q=2 p-2}\right\}$ be the vertices of $S\left(W_{p}\right)$ which subdivides the edges of $G$. Let $v_{0}$ be a single vertex in $W_{p}$, then $N\left[v_{0}\right]=\left\{w_{1}, w_{2}, w_{3}, \ldots, w_{p-1}, v_{0}\right\}$. Now, the nonsplit dominating set of $S\left(W_{p}\right)$ is $\left(\left\{v_{0}, v_{1}, \ldots, v_{p-1}, w_{1}, w_{2}, w_{3}, \ldots, w_{q}\right\}-\right.$ 
$\left.\left\{w_{1}, w_{2}, w_{3}, \ldots, w_{p-1}, v_{0}\right\}\right) \cup\left\{w_{i}\right\}$ where $w_{i} \in N\left(v_{0}\right)$. That is, $|D| \leq p+q-$ $p+1$. Hence, $\gamma_{n s}\left(S\left(W_{p}\right)\right) \leq q+1$.

Also, let $D_{0}$ be a minimum nonsplit dominating set of the cycle $\left\{v_{1}, w_{p}\right.$, $\left.v_{2}, w_{p+1}, \ldots, v_{p-1}, w_{2 p-2}, v_{1}\right\}$. Then $\left|D_{0}\right|=2 p-2=2(p-1)$, it follows from Proposition 2.1. Thus $D$ has at least one vertex other than the cycle which is adjacent to a vertex of the cycle. Now, the nonsplit dominating set is $\left\{v_{1}, w_{p}, v_{2}, w_{p+1}, \ldots, v_{p-1}, w_{2 p-2}\right\} \cup\left\{w_{k}\right\}$ where $1 \leq k \leq p-1$. That is,

$$
\begin{aligned}
D & =D_{0} \cup\left\{w_{k}\right\} \\
|D| & \geq\left|D_{0}\right|+1 \\
& \geq 2(p-1)+1 \\
|D| & \geq q+1 .
\end{aligned}
$$

Thus $\gamma_{n s}\left(S\left(W_{p}\right)\right)=q+1$.

Theorem 2.4. For any complete graph $K_{p}$ with $p \geq 2$ vertices, $\gamma_{n s}\left(S\left(K_{p}\right)\right)=$ $q+1$.

Proof. Let $G$ be a complete graph. Let $V(G)=\left\{v_{0}, v_{1}, v_{2}, \ldots, v_{p-1}\right\}$ be the vertices of $G$. Let $W=\left\{w_{1}, w_{2}, w_{3}, \ldots, w_{q}\right\}$ be the vertices of $S\left(K_{p}\right)$ which subdivides the edges $e_{i}(1 \leq i \leq q)$. Let $v_{0}$ be a vertex in $K_{p}$, then $N\left[v_{0}\right]=\left\{w_{1}, w_{2}, w_{3}, \ldots, w_{p-1}, v_{0}\right\}$. For some $w_{i} \in N\left(v_{0}\right)$, the nonsplit dominating set of $G$ is $\left\{v_{0}, v_{1}, v_{2}, \ldots, v_{p-1}, w_{1}, w_{2}, \ldots, w_{q}\right\}-\left\{w_{1}, w_{2}, w_{3}, \ldots, w_{p-1}, v_{0}\right\} \cup$ $\left\{w_{i}\right\}$. Hence $\gamma_{n s}\left(S\left(K_{p}\right)\right) \leq q+1$.

Also, any nonsplit dominating set of $S\left(K_{p}\right)$ contains at least $p-1$ vertices from $K_{p}$ and at least $q-p+2$ vertices from $V\left(S\left(K_{p}\right)\right)-V\left(K_{p}\right)$, so that

$$
\begin{aligned}
& \gamma_{n s}\left(S\left(K_{p}\right)\right) \geq p-1+q-p+2 \\
& \gamma_{n s}\left(S\left(K_{p}\right)\right) \geq q+1
\end{aligned}
$$

Hence, $\gamma_{n s}\left(S\left(K_{p}\right)\right)=q+1$. 
Corollary 2.5. If $G$ be any complete graph, $\gamma_{n s}(S(G))-\gamma_{n s}(G)=q$.

Proof. It follows from Theorem 1.2 and Theorem 2.4.

Theorem 2.6. For any complete bipartite graph $K_{m, n}$ with $2 \leq m \leq n$, $\gamma_{n s}\left(S\left(K_{m, n}\right)\right)=m(n+1)$.

Proof. Let $V_{1}=\left\{u_{1}, u_{2}, u_{3}, \ldots, u_{m}\right\}$ and $V_{2}=\left\{v_{1}, v_{2}, v_{3}, \ldots, v_{n}\right\}$ be a bipartition of $K_{m, n}$. Let $W=\left\{w_{i j}: 1 \leq i \leq m, 1 \leq j \leq n\right\}$ be the vertices of $S\left(K_{m, n}\right)$ which is adjacent to $u_{i}$ and $v_{j}$ and also $S\left(K_{m, n}\right)$ has $p=m+n$ vertices and $q=m \times n$ edges. For some $u_{i} \in V_{1}$, $N\left[u_{i}\right]=\left\{w_{i 1}, w_{i 2}, w_{i 3}, \ldots, w_{i n}, u_{i}\right\}$. The nonsplit dominating set of $S(G)$ is $\left(\left\{u_{1}, u_{2}, u_{3}, .\right.\right.$.

., $\left.\left.u_{m}, v_{1}, v_{2}, v_{3}, \ldots, v_{n}\right\}-\left\{w_{i 1}, w_{i 2}, w_{i 3}, \ldots, w_{i n}, u_{i}\right\}\right) \cup\left\{w_{i j}\right\}$, for some $w_{i j} \in$ $N\left(u_{i}\right)$ that is,

$$
\begin{aligned}
|D| & \leq p+q-n-1+1 \\
& =m+n+m n-n \\
& =m n+m \\
\gamma_{n s}(S(G)) & \leq m(n+1) .
\end{aligned}
$$

Also, any nonsplit dominating set of $S\left(K_{m, n}\right)$ contains at least $n+(m-$ $1)$ vertices from $K_{m, n}$ and at least $(m-1) n+1$ vertices from $V\left(S\left(K_{m, n}\right)\right)-$ $V\left(K_{m, n}\right)$, so that

$$
\begin{aligned}
\gamma_{n s}\left(S\left(K_{m, n}\right)\right) & \geq n+(m-1)+(m-1) n+1 \\
= & n+m-1+m n-n+1 \\
= & m n+m \\
\gamma_{n s}(S(G)) \geq & m(n+1) .
\end{aligned}
$$

Hence, $\gamma_{n s}\left(S\left(K_{m, n}\right)\right)=m(n+1)$. 
Theorem 2.7. For any graph $G, \gamma_{n s}(S(G))=p+q-\Delta(G)$.

Proof. Let $V=\left\{v_{1}, v_{2}, v_{3}, \ldots, v_{p}\right\}$ be a vertices of $G$ and let $W=$ $\left\{w_{1}, w_{2}, w_{3}\right.$,

$\left.\ldots, w_{q}\right\}$ be the vertices of $S(G)$ which subdivides the edges $e_{i}(1 \leq i \leq$ $q)$. Let $v_{i}$ be a vertex of maximum degree $\Delta(G)$. Then $v_{i}$ dominates $N\left[v_{i}\right]$ and the vertices in $V(S(G))-N\left[v_{i}\right]$ dominate themselves. Hence, $\left(V(S(G))-N\left[v_{i}\right]\right) \cup\left\{w_{j}\right\}$ where $w_{j} \in N\left(v_{i}\right)$ is a nonsplit dominating set of cardinality $p+q-\Delta(G)$ and so $\gamma_{n s}(S(G)) \leq p+q-\Delta(G)$.

Now, let $D$ be any nonsplit dominating set of $S(G)$. Let $v_{1}$ and $w_{1}$ are any two adjacent vertices in $S(G)$.

Case $(i)$ :

If $w_{1} \in V-D$, then $v_{1} \in V-D$ or $v_{1} \in D$. Suppose $v_{1} \in V-D$. Clearly $v_{1}=v_{i}, v_{i}$ is dominated by any one adjacent vertex $w_{j}$ where $j \neq 1$. Suppose $v_{1} \in D, w_{1}$ dominates $v_{1}$ and $v_{i}$. Also $v_{1}$ dominates $w_{1}$. So $v_{i}$ is dominated by any one vertex of $w_{j}$ where $j \neq 1$. Thus, $V-D \leq N\left[v_{i}\right]-\left\{w_{j}\right\}$ is connected in $S(G)$.

Case $(i i)$ :

If $w_{1} \in D$, then $v_{1} \in D$ or $v_{1} \in V-D$. Suppose $v_{1} \in V-D$. Clearly $v_{1}=v_{i}$, since $w_{1}$ and $v_{1}$ are adjacent vertices. Thus, $V-D \leq N\left[v_{i}\right]-\left\{w_{1}\right\}$ is connected in $S(G)$. Suppose $v_{1} \in D, w_{1}$ is adjacent to $v_{i}$ or $w_{1}$ is not adjacent to $v_{i}$.

Subcase $(i)$ :

Suppose $w_{1}$ is adjacent to $v_{i}$. Clearly, $w_{1}$ dominates $v_{i}$. Then $V-D \leq$ $N\left[v_{i}\right]-\left\{w_{1}\right\}$ is the connected dominating set of $S(G)$.

Subcase $(i i)$ :

Suppose $w_{1}$ is not adjacent to $v_{i}$. Clearly, $v_{1}, w_{1} \in D$. Then $v_{i}$ is dominated by any one adjacent vertex $w_{k}$ in $D$, where $w_{k} \in N\left(v_{i}\right)$ and $k \neq 1$ Thus, $V-D \leq N\left[v_{i}\right]-\left\{w_{k}\right\}$ is connected.

From case (i) and case (ii), we get

$$
\begin{aligned}
& V-D \leq N\left[v_{i}\right]-\{w\} \text { where } w \in N\left(v_{i}\right) \\
& |V-D| \leq \Delta(G)
\end{aligned}
$$




$$
\begin{aligned}
|V|-|D| & \leq \Delta(G) \\
|D| & \geq|V|-\Delta(G) \\
\gamma_{n s}(S(G)) & \geq|V(S(G))|-\Delta(G) \\
\gamma_{n s}(S(G)) & \geq p+q-\Delta(G) .
\end{aligned}
$$

Thus, $\gamma_{n s}(S(G))=p+q-\Delta(G)$.

Corollary 2.8. If $G$ is any connected graph, then

(i) $\gamma_{n s}(S(G))+\gamma_{n s}(G) \leq 2 p+q-\Delta(G)-\omega(G)+1$ and

(ii) $\gamma_{n s}(S(G))-\gamma_{n s}(G) \geq q-\Delta(G)+\omega(G)+1$.

Proof. It follows from Theorem 1.4 and Theorem 2.7.

Theorem 2.9. If $G K_{2}$ is any graph with $\delta(G) \geq 1, \gamma_{n s}(\overline{S(G)})=2$.

Proof. Let $G K_{2}$ is any graph with $\delta(G) \geq 1$. Let $W=\left\{w_{1}, w_{2}, w_{3}, \ldots, w_{q}\right\}$ be a subdivision vertices. Let $u$ and $v$ be any two adjacent vertices in $G$ and that is not adjacent in $S(G)$. Let $w_{1}$ subdivide $u$ and $v$ in $S(G)$. Clearly, $w_{1}$ dominates $V(\overline{S(G)})-\{u, v\}$ vertex set in $\overline{S(G)}$. That is, $N\left[w_{1}\right]=$ $V(\overline{S(G)})-\{u, v\}$. Now $\left\{w_{1}\right\}$ is a minimum nonsplit dominating set of $\overline{S(G)}-\{u, v\}$. Thus, $\gamma_{n s}(\overline{S(G)}-\{u, v\})=1$. Also, $u$ and $v$ are adjacent in $\overline{S(G)}$. Thus, $\gamma_{n s}(\overline{S(G)})=2$.

Corollary 2.10. Let $G$ be any graph with $\delta(G) \geq 1, \gamma_{n s}(S(G))+\gamma_{n s}(\overline{S(G)})=$ $p+q-\Delta(G)+2$.

Proof. It follows from Theorem 2.7 and Theorem 2.9.

Theorem 2.11. Let $G$ be any $(p, q)$ graph having components $G_{1}, G_{2}, G_{3}, \ldots, G_{k}$,

(i) if $\delta(G) \geq 1$, then $\gamma_{n s}(\overline{S(G)})=2$ and

(ii) if $G$ has isolated vertices, then $\gamma_{n s}(\overline{S(G)})=1$. 
Proof. Let $G$ be any graph having components $G_{1}, G_{2}, G_{3}, \ldots, G_{k}$.

(i) It follows from Theorem 2.9.

(ii) Suppose $G$ has isolated vertex for one or more components. Clearly, $\gamma_{n s}(\overline{S(G)}) \geq 1$. Let $v$ be any one isolated vertex in $G$. Then, $v$ dominates all other vertices in $\overline{S(G)}$. That is, $N[v]=V(\overline{S(G)})$. Thus, the nonsplit dominating set of $\overline{S(G)}$ contains at most one isolated vertex from $G_{1}, G_{2}, G_{3}, \ldots, G_{k}$, so that $\gamma_{n s}(\overline{S(G)}) \leq 1$. Hence, $\gamma_{n s}(\overline{S(G)})=1$.

Corollary 2.12. Let $G$ be any graph with isolated vertices, $\gamma_{n s}(S(G))+$ $\gamma_{n s}(\overline{S(G)})=p+q-\Delta(G)+1$.

Proof. It follows from Theorem 2.7 and Theorem 2.11.

\section{References}

[1] F. Harary, Graph theory. Reading, MA: Addision-Wesley, 1969.

[2] T. W. Haynes, S. T. Hedetniemi, and P. J. Slater, Fundamentals of domination in graphs. New York, NY; Marcel Dekker, Inc., 1998.

[3] T. W. Haynes, S. M. Hedetniemi, S. T. Hedetniemi, D. P. Jacobs, J. Knisely, and L. C. Van Der Merwe, "Domination subdivision in numbers", Discussiones mathematicae graph theory, vol. 21, no. 2, pp. 239-253, 2001. doi: $10.7151 /$ dmgt.1147

[4] V. R. Kulli and B. Janakiram, "The nonsplit domination number of a graph", Indian Journal pure applications mathematical, vol. 31, no. 5, pp. 545-550, May 2000. [On line]. Available: https:/ / bit.ly/ 3lJtJwi 